\title{
IDENTIFICAÇÃO DE ADULTERANTES BENZODIAZEPÍNICOS EM MEDICAMENTOS FITOTERÁPICOS
}

\author{
Bianca Sant'ana Verly, Angélica Augusta Grigoli Dominato \\ Universidade do Oeste Paulista - UNOESTE, Curso de Biomedicina, Presidente Prudente, SP. e-mail: \\ verly.bianca@gmail.com
}

\section{RESUMO}

Os benzodiazepínicos estão entre as drogas mais prescritas no mundo, são medicamentos com ações sedativas hipnóticas, ansiolíticas, relaxantes musculares ou anticonvulsivantes. Embora sejam medicamentos considerados seguros, os efeitos colaterais podem surgir. Os fitoterápicos são medicamentos obtidos empregando-se, como principio ativo, exclusivamente, o produto extraído de vegetais. Seu mau uso pode ocasionar problemas à saúde que podem levar a internações hospitalares e, até mesmo a morte, devido a ocorrência de efeitos colaterais e tóxicos. Alguns fitoterápicos como Passiflora incarnata L., Piper methysticum Forst (Kava- Kava) e Crataegus oxyacantha, apresentam as mesmas ações dos benzodiazepínicos, como ansiolíticos e sedativos. O estudo teve como objetivo detectar a presença de benzodiazepínicos em amostras de medicamentos fitoterápicos comercializados em farmácias no município de Presidente Prudente (SP). O método utilizado foi cromatografia em camada delgada (CCD), que após a extração e concentração do extrato foi aplicado em placas cromatográficas contra os padrões de benzodiazepínicos. Dentre as 5 amostras estudadas, os resultados encontrados foram negativos para o padrão clonazepan. Somente um medicamento apresentou resultado positivo para diazepan e flurazepan. Neste contexto é necessária maior rigidez na fiscalização durante a produção e comercialização dos medicamentos fitoterápicos, para garantir ao consumidor produtos isentos de contaminantes capazes de causar dependência química.

Palavras-chave: benzodiazepínicos, medicamentos, fitoterápicos, efeitos colaterais, contaminantes

\section{IDENTIFICATION OF BENZODIAZEPINES ADULTERANTS IN PHYTOTHERAPICS REMEDIES}

\begin{abstract}
Benzodiazepines are among the most precribed remedies in the world, they are remedies with hypnotic, anxiolytic, muscle relaxants and anticonvulsants actions. Although they are considered safe drugs, the colateral effects may rise. Phytotherapics are remedies that are obtained employing as active principle, exclusively, the product extracted from plants. Their misuse can cause health problems that can lead to hospitalization and even death, because to the occurrence of colateral and toxic effects. Some phytotherapics like Passiflora incarnata L., Piper methysticum Forst (Kava- Kava) e Crataegus oxyacantha have the same actions of benzodiazepines as anxiolytics and sedatives. The study aimed to detect the presence of benzodiazepines in samples of phythoterapics remedies sold in pharmacies in the city of Presidente Prudente (SP). The method used was thin layer chromatography (TLC), which after extraction and concentration of the extract was applied to chromatographic plates against the standards of benzodiazepines. Among the five samples studied, the results were negative for clonazepam standard. Only one drug showed positive result for diazepam and flurazepan. In this context it is necessary more rigid supervision during the production and marketing of phytotherapics, to ensure consumer products free of contaminants capable of causing addiction.
\end{abstract}

Keywords: benzodiazepines, remedies, phytotherapics, colateral effects, contaminants 
INTRODUÇÃO

O consumo do benzodiazepínico aumentou com o passar do tempo, atualmente se estima o dobro de vendas a cada cinco anos. Esse aumento pode ser causado pela modificação da vida cotidiana das pessoas que não possuem mais tolerância de viver sob tanto estresse, ou também pelo aumento de propagandas por parte das indústrias farmacêuticas, além da prescrição, muitas vezes inadequadas dos médicos ${ }^{1}$.

Os fármacos ansiolíticos, como os benzodiazepínicos, são uns dos mais utilizados $^{1}$, por causa de sua alta eficácia e relativa segurança ${ }^{2}$. Eles funcionam como depressor do Sistema Nervoso Central. Tem como ações principais sensação de calma e sonolência, sendo considerados sedativohipnóticos. Sua utilização data da década de 1960, com o clordiazepóxido, conhecido popularmente como diazepam ${ }^{3}$.

Os benzodiazepínicos atuam nos receptores de neurotransmissores inibitórios ativados pelo GABA, é nessa ação que se deve o surgimento de efeitos sedativos e hipnóticos dessa classe de medicamentos, mas que também pode causar dependência ${ }^{3}$. Os sintomas relacionados à abstinência incluem: ansiedade, agitação, irritabilidade, insônia, cefaléia, tremores, tontura, anorexia, náusea, vômitos, diarreia, fraqueza, fotofobia, despersonalização e depressão ${ }^{2}$.

Esses efeitos adversos geraram uma motivação para buscar novas fontes alternativas de tratamento, com isso o uso de plantas medicinais, ou seja, os fitoterápicos surgiram como uma opção de maior risco/benefício em relação aos medicamentos sintéticos comercializados ${ }^{4}$.

A fitoterapia se resume em utilizar plantas medicinais com fins medicamentosos, pois, além de ser utilizada como uma forma alternativa de tratamento de uma doença, também é vista de uma maneira a desenvolver a saúde com finalidade preventiva ${ }^{5}$. É possível observar o aumento da comercialização de produtos fitoterápicos em todo o mundo e no Brasil. A motivação pode estar relacionado à ampla divulgação pelos meios de comunicação, pouco rigor na fiscalização, assim como a facilidade de aquisição e, no caso do Brasil, a dificuldade da população em ter acesso a um bom acompanhamento médico e o alto custo de medicamentos industrializados ${ }^{6}$.

Apesar dos fitoterápicos serem considerados medicamentos naturais, sua utilização também deve ser monitorada, principalmente porque existe uma falsa concepção da população em geral, de que esses medicamentos não causam danos à saúde, levando assim a um grande número 
de casos de intoxicação. Os princípios ativos de plantas podem produzir efeitos colaterais, ou tóxicos, ainda não descritos na literatura ${ }^{7}$.

Segundo Veiga Jr et al. ${ }^{8}$, merece atenção a existência da presença de adulterantes que são adicionados à composição dos fitoterápicos, visando aumentar os efeitos propagados. Essa atividade é ilegal e constitui uma violação grave da Lei da Vigilância Sanitária, sendo que as classes mais frequentes de medicamentos encontradas nas formulações fitoterápicas são anorexígenos, benzodiazepínicos, antidepressivos e hipoglicêmicos ${ }^{9}$.

Segundo Santos et al. $^{10}$, adição de benzodiazepínicos em medicamentos comercializados como fitoterápicos, além de ser proibida, é preocupante, pois podem ocorrer interações medicamentosas entre as duas substâncias. A interação medicamentosa trata-se da interferência no efeito do medicamento, com outro medicamento, alimento ou até mesmo bebidas alcoólicas.

Os fitoterápicos podem reagir com outras substâncias, causando o aumento ou diminuição dos seus efeitos, ou até mesmo provocando reações tóxicas ao organismo ${ }^{5}$. No caso da interação dos fitoterápicos com os benzodiazepínicos, ocorre o sinergismo entre essas duas substâncias, ou seja, os efeitos metabólicos aumentam ${ }^{8}$.
A regulamentação em vigor para o registro de medicamentos fitoterápicos e a Resolução de Diretoria Colegiada (RDC) 48/2004, que vem determinar alguns aspectos essenciais que devem compor o registro, como a identificação botânica das espécies vegetais ali utilizadas, padrão de qualidade e identidade e provas de eficácia e seguranças que validem as indicações terapêuticas ali propostas ${ }^{11}$.

Por se tratar de algo que pode colocar em risco a vida, a identificação dos adulterantes em formulações fitoterápicas é de grande importância. Existem diferentes métodos para identificação destas substâncias. Um dos métodos mais utilizados para o estudo de adulteração é a cromatografia em camada delgada, por ser um procedimento analítico rápido, de baixo custo e abrangente $\mathrm{e}^{12}$.

O objetivo deste estudo foi identificar a presença de benzodiazepínicos em amostras de medicamentos fitoterápicos popularmente conhecidos e de baixo custo.

\section{METODOLOGIA}

Foram escolhidos cinco medicamentos fitoterápicos de marcas diferentes que possuem as mesmas ações dos benzodiazepínicos, utilizados como ansiolíticos e hipnóticos, contendo os princípios ativos da Piper methysticum, Passiflora incarnata, Crataegus oxycantha, 
Salix alba, Passiflora alata e Erythrina mulungu.

As amostras foram enumeradas de $1 \mathrm{a}$ 5, sendo as amostras 1, 2 e 3 apresentavam a forma de comprimido, a amostra 4 de cápsula e a amostra 5 estava em forma líquida. O procedimento foi realizado em triplicata para cada tipo de fitoterápico.

As amostras por apresentarem diferentes formas farmacêuticas foram manipuladas de formas diferentes para que os princípios ativos pudessem ser extraídos eficientemente. Os medicamentos, em comprimido, foram triturados com o auxílio de um almofariz, em seguida foram adicionados em $15 \mathrm{~mL}$ de água destilada. $\mathrm{A}$ amostra na forma farmacêutica de cápsula, foram abertas e o conteúdo foi adicionado em $15 \mathrm{~mL}$ de água. O medicamento líquido foi utilizado o volume de $15 \mathrm{~mL}$ para o procedimento de extração.

Após a solubilização das amostras sólidas em água destilada, foi realizado o processo de extração dos agentes tóxicos (benzodiazepínicos). Em funil de separação, foi transferido todo o volume de cada amostra, e adicionado $30 \mathrm{~mL}$ do agente extrator (clorofórmio:isopropanol (95:5)), com agitação vigorosa durante 1 minuto. Após a separação das fases, a orgânica foi filtrada em sulfato de sódio anidro e concentrada totalmente, ou seja até completa evaporação do agente extrator, obtendo-se o extrato. Para o procedimento de identificação preparou-se a placa para CCD, com ativação da mesma $\left(100{ }^{\circ} \mathrm{C} / 1 \mathrm{~h}\right)$ e limitação da corrida cromatográfica em 10 $\mathrm{cm}$. Os extratos foram solubilizados com acetona (p.a.) e aplicados, o volume de $10 \mu \mathrm{L}$, na placa de CCD, com o auxílio de um pipetador automático. Os padrões de benzodiazepínicos (1 $\mathrm{mg} / \mathrm{mL}$ ) utilizados foram diazepam, flurazepam e clorazepam. As placas estiveram, em cuba cromatográfica contendo como fase móvel clorofórmio:acetona (9:1). A seguir, foi realizada a revelação com solução de Dragendorf iodado (revelador universal), as manchas das amostras foram comparadas com as dos padrões e foi calculado o Rf, e a porcentagem de erro. Nos casos positivos a porcentagem estava menor que $5 \%{ }^{13}$.

\section{RESULTADOS}

Dos cinco medicamentos fitoterápicos estudados, quatro demonstraram resultados negativos (amostras 1, 2, 3 e 4) para a presença de benzodiazepínicos para a técnica de $C C D$, enquanto que a amostra 5 apresentou resultado positivo para diazepam e flurazepam (Tabela 1).

As manchas formadas, após o uso do revelador universal, na amostra 5, foram comparadas com os padrões (diazepam e flurazepan), e estiveram semelhantes tanto no formato, coloração como com os Rf. De 
acordo com o cálculo do $\mathrm{Rf}$ os resultados estavam positivos por que apresentaram porcentagens de erro média de 2,3\%, enquanto que para o flurazepam apresentou $0,0 \%$ de erro, pois os Rf calculados estiveram iguais, entre amostra e padrão.

Tabela 1. Resultados das 5 amostras de medicamentos fitoterápicos, após a identificação pelo método de cromatografia em camada delgada, contra os padrões da classe farmacológica benzodiazepínicos.

\begin{tabular}{l|c|c|c|c|c}
\hline \multicolumn{1}{c|}{ Amostra } & 1 & 2 & 3 & 4 & 5 \\
\hline Padrões \\
\hline Diazepam & negativo & negativo & Negativo & negativo & Positivo \\
\hline Flurazepam & negativo & negativo & Negativo & negativo & Positivo \\
\hline Clorazepam & negativo & negativo & Negativo & negativo & negativo \\
\hline
\end{tabular}

\section{DISCUSSÃO}

Silveira et al. $^{6}$ relataram que o consumo de medicamentos fitoterápicos pode estar relacionado com a ampla divulgação na mídia, dificuldade do brasileiro ao acesso e acompanhamento de médicos durante o tratamento, além da facilidade de aquisição dos fitoterápicos. Com isso cresce a preocupação com o uso indiscriminado destes tipos de medicamentos, que podem causar doenças iatrogênicas e/ou intoxicações.

Segundo Lima ${ }^{5}$, os métodos analíticos possuem importante papel na identificação da presença de adulterantes em formulações fitoterápicas. Existem diversas metodologias para análise de adulteração em materiais biológicos ou não, como a cromatografia gasosa acoplada a espectrometria de massas (GC-MS), cromatografia líquida de alta eficiência com espectrometria de massas
(HPLC-MS), cromatografia líquida de alta eficiência com detector de UV (HPLC-UV), cromatografia gasosa acoplada com espectrometria de massa (CG-MS), eletroforese capilar (CE).

O método de cromatografia em camada delgada foi utilizado por Azeredo et al. ${ }^{12}$ para identificação de adulteração em fitoterápicos, semelhante a esse estudo, assim como Yano et al. ${ }^{14}$, demonstraram que entre as 22 amostras utilizadas, três delas foram consideradas insatisfatórias por conter anorexígenos e diazepam na formulação, sem estar declarado no rótulo.

\section{CONCLUSÃO}

$\mathrm{O}$ resultado positivo para diazepan e flurazepan em um medicamento estudado, estabelece a necessidade de maior rigidez na fiscalização de indústrias de fitoterápicos, a fim de controlar a presença destes 
adulterantes nos produtos. Laboratórios de análises de medicamentos credenciados pela Agência Nacional de Vigilância Sanitária (ANVISA) e Ministério da Saúde (MS) analisam os medicamentos comercializados apreendidos após fiscalização. Porém esta prática deve ser realizada com maior frequência, para assim minimizar os riscos de consumo de fitoterápicos adulterados durante sua produção industrial. Assim a atuação entre as agências reguladoras e campanhas educativas pode minimizar os riscos de uso de medicamentos fitoterápicos contendo adulterantes capazes de causar dependência química.

\section{REFERÊNCIAS}

1. Auchewski I, Andreatini R, Galduroz JCF, Lacerda RB. Avaliação da orientação médica sobre os efeitos colaterais de benzodiazepínicos. Rev Bras Psiquiatr. 2004;26(1):24-31.

2. Silva P. Farmacologia. 8ed. Editora Guanabara Koogan: São Paulo; 2010.

3. Foscarini PT. Benzodiazepínicos: uma revisão sobre o uso, abuso e dependência. [Trabalho de Conclusão de Curso]. Universidade Federal do Rio Grande do Sul Porto Alegre, 2010.

4. Provensi, G. Investigação da atividade ansiolítica de Passiflora alata Curtis (Passifloracea). [Dissertação de Mestrado]. Universidade Federal do Rio Grande do Sul Porto Alegre, 2007.
5. Lima APS. Desenvolvimento de métodos eletroforéticos e cromatográficos para a determinação de benzodiazepínicos como adulterantes em formulações fitoterápicas para emagrecimento. [Dissertação de Mestrado]. Universidade Federal de Santa Maria Rio Grande do Sul, 2009.

6. Silveira PF, Bandeira MAM, Arrais PSD. Farmacovigilância e reações adversas às plantas medicinais e fitoterápicos: uma realidade. Rev Bras Farmacognosia. 2008;18(4):618-626.

7. Nicoletti MA, Oliveira-Júnior MA, Bertasso CC, Caporossi PY, Tavares APL. Principais interações no uso de medicamentos fitoterápicos. Infarma. 2007;19(1):32-40.

8. Veiga Junior VF, Pinto AC, Maciel MAM. Plantas medicinais: cura segura? Química Nova. 2005;28(3):519-528.

9. Correia D. Determinação voltamétrica de 1-4 benzodiazepínico e dietilpropiona como adulterantes em fitoterápicos para emagrecimento. [Dissertação de Mestrado]. Universidade Federal de Santa Maria Rio Grande do Sul, 2008.

10. Santos $H C$, Ribeiro RR, Ferrarini $M$, Fernandes JPS. Possíveis interações medicamentosas com psicotrópicos encontradas em pacientes da Zona Leste de São Paulo. Rev Ciên Farmacêuticas Básica Aplic. 2009;30(3):285-289.

11. Carvalho ACB, Balbino EE, Maciel $A$, Perfeito JPS. Situação do registro de medicamentos fitoterápicos no Brasil. Rev Bras Farmacognosia. 2008;18(2):314-319.

12. Azeredo FS, Guimarães RI, Paula JR, Cunha IC. Validação de técnica analítica em cromatografia em camada delgada comparativa para identificação de fármacos anorexígenos sintéticos em produtos fitoterápicos. Rev Eletrônica Farmácia. 2004; (1): 17-24. 
13. Moraes ECF, Sznelwar RB, Fernicola NAGG. Manual de Toxicologia Analítica. Ed Roca, 1991.

14. Yano HM. Pesquisa de anorexígenos e benzodiazepínicos em formulações emagrecedoras e avaliação de rotulagem, em análises da Seção de Farmacognosia do Instituto Adolfo Lutz no período de junho de 2004 a março de 2007. Rev Inst Adolfo Lutz. 2008.

Recebido para publicação em 19/08/2014

Revisado em 24/08/2014

Aceito em 02/09/2014 